\title{
Modeling and Simulating a Battery for an Electric Vehicle Based on Modelica
}

\author{
Dongchen Qin ${ }^{1}$. Jianjie $\mathrm{Li}^{1}$ (1) · Tingting Wang ${ }^{1}$. Dongming Zhang ${ }^{1}$
}

Received: 4 March 2019 / Accepted: 5 August 2019 / Published online: 27 August 2019

(c) The Author(s) 2019

\begin{abstract}
Battery is the key technology to the development of electric vehicles, and most battery models are based on the electric vehicle simulation. In order to accurately study the performance of $\mathrm{LiFePO}_{4}$ batteries, an improved equivalent circuit model was established by analyzing the dynamic characteristics and contrasting different-order models of the battery. Compared to the traditional model, the impact of hysteresis voltage was considered, and the third-order resistance-capacitance (RC) network was introduced to better simulate internal battery polarization. The electromotive force, resistance, capacitance and other parameters were calibrated through battery charge and discharge experiments. This model was built by using Modelica, a modeling language for object-oriented multidomain physical systems. MWorks was used to implement the cycle conditions and vehicle simulation. The results show that the third-order RC battery model with hysteretic voltage well reflects the dynamics of a $\mathrm{LiFePO}_{4}$ battery. The difference between the simulated and measured voltages is small, with a maximum error of $1.78 \%$, average error of $0.23 \%$. The validity and feasibility of the model are verified. It can be used in unified modeling and simulation of subsequent multi-domain systems of electric vehicles.
\end{abstract}

Keywords Electric vehicle $\cdot \mathrm{LiFePO}_{4}$ battery $\cdot$ Hysteresis $\cdot$ Equivalent circuit model $\cdot$ Modelica

\section{Introduction}

An electric vehicle (EV) is a complex multi-domain physical system with mechanical, electronic and hydraulic components whose design needs coordinated modeling and simulation in multiple disciplines [1]. At present, there are two commonly used multi-domain modeling methods. One is the interface-based method. This method realizes co-simulation by using professional software in different fields and requires each software to have multi-domain interfaces. However, this leads to great difficulty in joint simulation when there are many design fields. The other method is directly using a unified multi-domain modeling language. This method can directly and clearly describe the coupling between physical systems in different domains, conveniently define interfaces and transfer information between system models in those domains. The simulation results are more reliable, so it has greater advantages and prospects in modeling multi-domain

Jianjie Li

1178787313@qq.com

1 School of Mechanical Engineering, Zhengzhou University, High-Tech Zone, 100 Science Avenue, New Campus of Zhengzhou University, Zhengzhou 450001, Henan, China systems. One example is the language Modelica [2], which has been used to model and simulate complex multi-domain physical systems in recent years. Its model library covers electrical, mechanical, thermal, control and many other characteristics, providing a basis for unified description of physical systems [3]. Moreover, Modelica supports objectoriented non-causal modeling, which has standardization, openness and extensibility. Modelica is more accurate and efficient for complex physical modeling [4].

The battery is essential for the power, safety and economy of an EV. A good design of the battery management system improves battery life and ensures cruising range and vehicle safety [5]. The battery model is used to describe the dynamics of battery operation. The model is indispensable to estimate the battery state of charge (SOC) and simulate the battery management system of an EV. It is difficult to model and simulate the battery management process [6]. Generally, a battery model is divided into an electrochemical model, artificial neural network model and equivalent circuit model. The electrochemical model describes the chemical reactions at the molecular level inside the battery, and examples are the Shepherd and Unnewehr models [7, 8]. The dynamic behavior of the battery is described by ordinary differential equations for each reaction. To attain proper accuracy with 
the model, many parameters are used to simulate the battery polarization. Because the electrochemistry of the battery is related to the environmental conditions, it is too complicated to achieve an accurate electrochemical model. Even if a more accurate model can be established under certain conditions, its application to actual working conditions is still limited [9]. An artificial neural network model makes full use of the nonlinear and self-learning characteristics of a neural network and combines experimental data to establish the relationships among various parameters of the battery system. Examples are the BP network and radial basis network [10]. The disadvantage is that a network needs a large amount of experimental data to predict the battery performance. The equivalent circuit model uses the resistance, capacitance, constant voltage source and other circuit elements to simulate battery dynamics. Commonly used equivalent circuit models include the Rint, RC, PNGV (Partnership for a New Generation of Vehicles) and Thevenin models [11]. The RC model only describes the battery polarization using the capacitance and does not reflect the resistance. The PNGV model is a standard model that simulates the complex internal relationships of the battery during charging and discharging, but the complexity of its algorithm makes simulation difficult. The Thevenin model reflects the internal capacitance and resistance of the battery, and its algorithm is relatively simple and easy to implement.

MWorks is a relatively mature simulation platform of Modelica in China. However, its library only has a model for static battery internal resistance and no model that can well describe the dynamic performance of a battery. Zhang et al. [12] established the Simulink model of the second-order RC equivalent circuit. The simulation error based on the Federal Urban Driving Schedule was less than 5\%. Huang et al. [13] used Modelica to model the PNGV equivalent circuit with a simulation error of less than $2 \%$ relative to bench test results.

Hysteresis can be found according to the mismatch of the charge-discharge equilibrium potential curve of the $\mathrm{LiFePO}_{4}$ battery. Hysteresis can be found according to the mismatch of the charge-discharge equilibrium potential curve of the $\mathrm{LiFePO}_{4}$ battery [14-16]. However, previous research has rarely considered the hysteresis voltage of the $\mathrm{LiFePO}_{4}$ battery. To eliminate the simulation error it generates, the mathematical relationship between hysteretic voltage, electromotive force (EMF) and SOC was established in this study. The overpotential errors of different-order RC networks were analyzed, and a more suitable third-order RC network was selected to fit the battery polarization. Compared with previous models, an improved third-order RC battery model with hysteresis voltage was established by using Modelica. The cycle conditions and vehicle simulation were implemented on MWorks. The validity and practicability of the model were fully verified, and it provides more effective unified modeling and simulation of the multi-domain system of the EV.

\section{Dynamic Performance Analysis of a LiFePO ${ }_{4}$ Battery}

An EV battery is affected by environmental temperature, degree of aging and complex internal chemical reactions. Its characteristics are different under different external conditions, driving conditions and charging and discharging processes. The dynamics of $\mathrm{LiFePO}_{4}$ batteries can be divided into two aspects: EMF and overpotential. These are described separately in the following sections.

\subsection{Electromotive Force Characteristic Analysis}

EMF refers to the potential difference between positive and negative electrodes when the whole battery system is in equilibrium. The equilibrium potential curves $(\mathrm{OCV}-\mathrm{SOC}$ curves) for charging and discharging of a $\mathrm{LiFePO}_{4}$ battery do not coincide, that is, hysteretic voltage exists [17]. To calculate the EMF, it is first necessary to obtain an experimental charge-discharge equilibrium potential curve. Then, using the electrochemical mechanism that produces hysteretic voltage, the EMF can be obtained by weighting the charge-discharge equilibrium potential:

Discharge: $\quad V_{\mathrm{H}}=\lambda\left(E_{\mathrm{c}}-E_{\mathrm{d}}\right)$

Charge: $\quad V_{\mathrm{H}}=(1-\lambda)\left(E_{\mathrm{c}}-E_{\mathrm{d}}\right)$

$\mathrm{EMF}=\lambda E_{\mathrm{c}}+(1-\lambda) E_{\mathrm{d}}$

The quantities $E_{\mathrm{d}}$ and $E_{\mathrm{c}}$ represent the equilibrium potentials of the battery discharge and charge, respectively. $\lambda$ is the weight, and $V_{\mathrm{H}}$ is the difference between the charge/ discharge equilibrium potential and EMF. Therefore, the equivalent voltage source can be modeled separately according to the EMF and $V_{\mathrm{H}}$.

\subsection{Overpotential Characteristic Analysis}

During the operation of a $\mathrm{LiFePO}_{4}$ power battery, the electrode reaction causes the electrode potential to deviate from the equilibrium potential, and the deviation value is the overpotential. The overpotential is mainly manifested in two aspects: the equivalent impedance of the battery and the rebound voltage [17].

The equivalent impedance causes a certain voltage drop when the current passes through the battery. This is externally represented by the equivalent internal resistance of the battery, which is mainly composed of two parts: ohmic resistance and polarization resistance. For a certain battery system, these can be considered to be constant within a particular temperature range and cycle. 
Table 1 Fitting errors of RC networks with different orders

\begin{tabular}{llll}
\hline Orders & Standard deviation $(\mathrm{V})$ & $\begin{array}{l}\text { Maximum } \\
\text { deviation }(\mathrm{V})\end{array}$ & $\begin{array}{l}\text { Average } \\
\text { deviation } \\
(\mathrm{V})\end{array}$ \\
\hline Second order & 0.0005751 & 0.00320 & 0.000225 \\
Third order & 0.0002144 & 0.00086 & 0.000163 \\
Fourth order & 0.0002313 & 0.00062 & 0.000115 \\
Fifth order & 0.0001398 & 0.00057 & 0.000101 \\
\hline
\end{tabular}

The rebound voltage is mainly related to the electrolyte conductivity. Because the electrolyte conductivity of a $\mathrm{LiFePO}_{4}$ battery is low, the electrolytes cannot replenish the lithium ions at a high discharge current, resulting in a voltage drop. When the battery stops discharging, the lithium ions undergo diffusion and phase transition, which causes the entire system to gradually return to equilibrium. The external performance is an orderly rise and fall of the open-circuit voltage, which is voltage rebound.

Generally, the overpotential of a $\mathrm{LiFePO}_{4}$ battery shows both resistance and capacitance, so it can be realized with an RC network model. As shown in Table 1, the Electric Vehicle Research Center of Sun Yat-sen University has computed the simulation errors of the open-circuit voltage (OCV) relative to the measured values when $\mathrm{RC}$ networks with different orders are applied to the $\mathrm{LiFePO}_{4}$ battery [17]. Because the calculated rebound characteristics of the first-order RC network are far from those of the actual battery, only the errors generated by the second- to fifth-order networks are compared. It can be seen in Table 1 that the higher the order of the RC network, the more accurate the fitting, but the corresponding model complexity is higher. Therefore, after consideration of all factors, a third-order RC network is chosen to represent the overpotential of the $\mathrm{LiFePO}_{4}$ battery.

\section{Analysis and Modeling of $\mathrm{LiFePO}_{4}$ Battery Model}

\subsection{Description of $\mathrm{LiFePO}_{4}$ Battery Model}

From the above analysis of $\mathrm{LiFePO}_{4}$ battery characteristics, the main dynamics considered in this paper include the equivalent voltage source reflecting the hysteresis voltage and the RC network reflecting the overpotential. [12]. A third-order RC battery model with hysteretic voltage was constructed as shown in Fig. 1. The equivalent voltage source $E_{\mathrm{B}}$ is composed of controlled voltage sources EMF and $V_{\mathrm{H}}$. The EMF source is controlled by $V_{\mathrm{SOC}}$, which represents the voltage of capacitance $C_{\mathrm{CAP}}$, and its value is equal to SOC. The capacitance $C_{\mathrm{CAP}}$ represents the rated capacity of the battery. The controlled source $V_{\mathrm{H}}$ represents

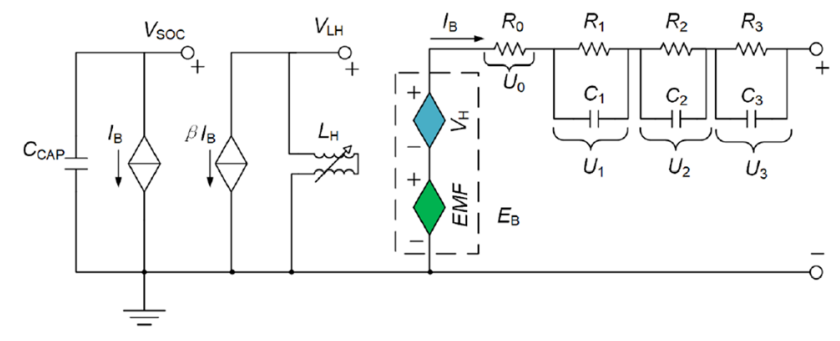

Fig. 1 Equivalent circuit model of third-order RC network for $\mathrm{LiFePO}_{4}$ battery

the battery hysteresis voltage, which is jointly controlled by $V_{\mathrm{SOC}}$ and the voltage $V_{\mathrm{LH}}$. The direction of $V_{\mathrm{H}}$ is controlled by the voltage $V_{\mathrm{LH}}$ across $L_{\mathrm{H}}$. The adjustable inductor $L_{\mathrm{H}}$ is used to judge whether the battery was previously charging or discharging.

As for the remaining quantities, $R_{0}$ represents the ohmic resistance. $R_{1}, R_{2}$ and $R_{3}$ are the polarization resistances, and $C_{1}, C_{2}$ and $C_{3}$ are the polarization capacitances. From Kirchhoff's law, the following mathematical relationships can be obtained for the equivalent circuit.

The formulation of the equivalent voltage source is as follows:

$\left\{\begin{array}{l}\mathrm{EMF}=f_{1}\left(V_{\mathrm{SOC}}\right) \\ V_{\mathrm{H}}=f_{2}\left(V_{\mathrm{SOC}}, V_{\mathrm{LH}}\right) \\ E_{\mathrm{B}}=\mathrm{EMF}+V_{\mathrm{H}}\end{array}\right.$

Here,

$V_{\mathrm{SOC}}=$ Initial $S O C+\frac{1}{C_{\mathrm{CAP}}} \int I_{\mathrm{B}} \mathrm{d} t$.

The formulation of the equivalent impedance is as follows:

$\left\{\begin{array}{l}C_{1} \cdot \frac{\mathrm{d} U_{1}}{\mathrm{~d} t}+\frac{U_{1}}{R_{1}}=I_{\mathrm{B}} \\ C_{2} \cdot \frac{\mathrm{d} U_{2}}{\mathrm{~d} t}+\frac{U_{2}}{R_{2}}=I_{\mathrm{B}} \\ C_{3} \cdot \frac{\mathrm{d} U_{3}}{\mathrm{~d} t}+\frac{U_{3}}{R_{3}}=I_{\mathrm{B}} \\ U_{0}=I_{\mathrm{B}} R_{0}\end{array}\right.$

The quantity $U_{0}$ is the terminal voltage of $R_{0} . U_{1}, U_{2}$ and $U_{3}$ are the terminal voltages of the $R_{1} C_{1}, R_{2} C_{2}$ and $R_{3} C_{3}$ series links, respectively. InitialSOC is the initial battery SOC, and the remaining parameters are consistent with those described above.

\subsection{Modelica Model of LiFePO ${ }_{4}$ Battery}

To establish a model that is consistent with a real vehicle, this study chose unified modeling based on Modelica to 


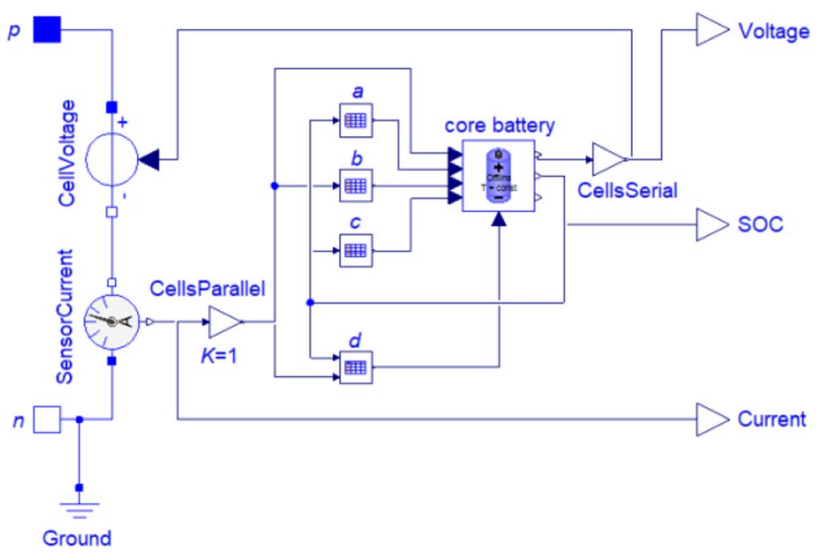

Fig. 2 Modelica model of $\mathrm{LiFePO}_{4}$ battery

construct complex systems relating different areas. Traditional modeling tends to simulate and analyze the performance of a single domain. Examples are MATLAB and Simulink, where models cannot directly reflect topological relationships. Multi-domain unified modeling based on Modelica is compatible with graph-based modeling and with object-oriented and equation-based non-causal features. Furthermore, it has better simulation effects, such as in MWorks.

According to Fig. 1 and Eqs. (4) and (5), the battery model is established using variable resistor, signal voltage, variable capacitor and other components in the Electrical Component Library of the Modelica Standard Library. In Fig. 2, components $a, b, c$ and $d$ are four table lookup modules, in which $a, b$ and $c$ represent the third-order RC network parameters of the battery model, and $d$ is the hysteretic voltage $V_{\mathrm{H}}$. The battery internal resistance and EMF-related parameters are encapsulated in the core battery module. The model uses current as input, and $p$ and $n$ are the input ports. Then, the voltage and SOC could be obtained as output data to verify the model, as shown in Fig. 2.

\subsection{Determination of Model Parameters}

\subsubsection{Parameter Estimation of Equivalent Voltage Source}

The weights are determined sequentially according to Eqs. (1)-(3) and the hysteretic voltage of the $\mathrm{LiFePO}_{4}$ battery [17]. The hysteresis voltage is relatively stable in the platform region $(\mathrm{SOC}=0.1 \sim 0.9)$, and the weight can be taken as $\lambda=0.5$ when calculating $V_{\mathrm{H}}$. In the non-platform region $(\mathrm{SOC}=0 \sim 0.1,0.9 \sim 1)$, the relationship between the SOC and the weight $\lambda$ is approximately linear. When $V_{\mathrm{SOC}}=0 \sim 0.1, \lambda=1 \sim 0.5$, and when $V_{\mathrm{SOC}}=0.9 \sim 1, \lambda=0.5 \sim 0$ [18].

In this study, the $\mathrm{LiFePO}_{4}$ battery provided by the project partner company was used for related experiments.
The rated voltage of the battery is $3.2 \mathrm{~V}$, and the rated capacity is $2.2 \mathrm{Ah}$. According to the manufacturer guidelines, when the battery is charged to $0.01 \mathrm{C}$ with a constant voltage of $3.7 \mathrm{~V}$ at $25 \pm 2{ }^{\circ} \mathrm{C}$, it is recorded as $100 \%$ SOC. When the battery is discharged to the cutoff voltage of $2.5 \mathrm{~V}$ with a constant current of $0.5 \mathrm{~A}$, it is recorded as $0 \%$ SOC. Subsequently, the battery SOC is adjusted by charging and discharging with a small current to a node where the battery SOC is $100 \%, 95 \%, 90 \% \ldots \ldots .10 \%, 5 \%$ and $0 \%$. The corresponding OCV is recorded separately after standing for an hour. Figure 3 shows the relationship between charge/discharge OCV and SOC for the $\mathrm{LiFePO}_{4}$ battery.

The charge/discharge curves in Fig. 3 do not completely overlap. This indicates hysteresis in the $\mathrm{LiFePO}_{4}$ battery. The hysteresis voltage $V_{\mathrm{H}}$ is determined by the electrochemical characteristics of the battery itself. The discharge equilibrium potential is always below the charge equilibrium potential at the same SOC. If the two potentials were not distinguished, the charge/discharge hysteresis voltage would cause a large error in the battery simulation and SOC estimation. Therefore, according to the charge/discharge potential curves and the electrochemical mechanism of the hysteresis voltage, the charge/discharge equilibrium potentials are weighted to obtain the battery EMF using Table 2 and Eq. (4).

As shown in Fig. 4, it is better to model EMF and $V_{\mathrm{H}}$ separately and then combine them to achieve accurate test results. In the actual modeling, the battery EMF can be looked up in a table of values obtained from the EMF--SOC curve.

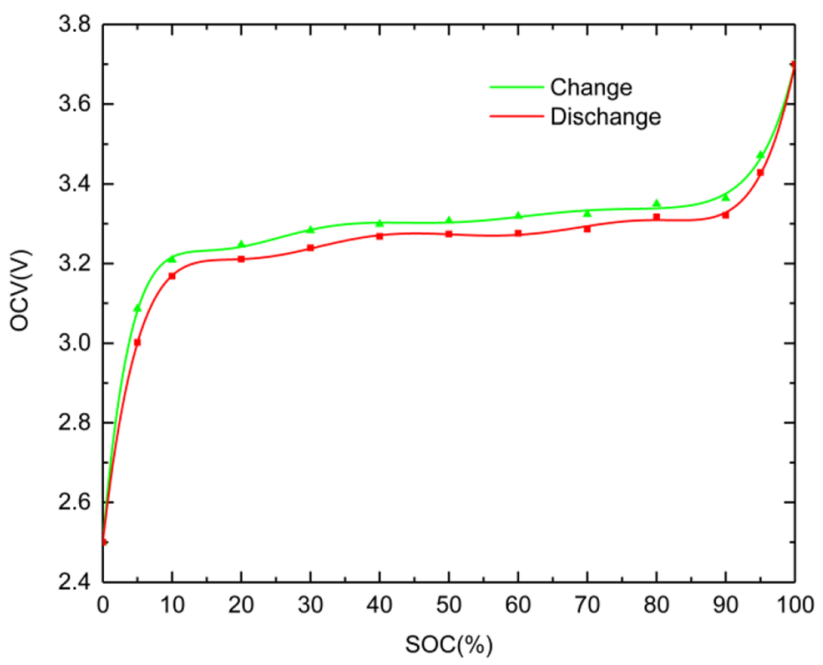

Fig. 3 Relationships between OCV and SOC in charging and discharging processes for the $\mathrm{LiFePO}_{4}$ battery 
Table 2 Formulas for calculating charge-discharge hysteresis voltage $V_{\mathrm{H}}$ and EMF for different SOCs

\begin{tabular}{llll}
\hline SOC & $V_{\mathrm{H}}$ (during charging $)$ & $V_{\mathrm{H}}($ during discharging $)$ & EMF \\
\hline $0-0.1$ & $V_{H}=5 \operatorname{SOC}\left(E_{\mathrm{c}}-E_{\mathrm{d}}\right)$ & $V_{\mathrm{H}}=(-5 \mathrm{SOC}+1)\left(E_{\mathrm{c}}-E_{\mathrm{d}}\right)$ & $\mathrm{EMF}=(-5 \mathrm{SOC}+1) E_{\mathrm{c}}+(5 \mathrm{SOC}) E_{\mathrm{d}}$ \\
$0.1-0.9$ & $V_{\mathrm{H}}=0.5\left(E_{\mathrm{c}}-E_{\mathrm{d}}\right)$ & $V_{\mathrm{H}}=0.5\left(E_{\mathrm{c}}-E_{\mathrm{d}}\right)$ & $\mathrm{EMF}=0.5\left(E_{\mathrm{c}}+E_{\mathrm{d}}\right)$ \\
$0.9-1$ & $V_{\mathrm{H}}=(5 \mathrm{SOC}-4)\left(E_{\mathrm{c}}-E_{\mathrm{d}}\right)$ & $V_{\mathrm{H}}=(-5 \mathrm{SOC}+5)\left(E_{\mathrm{c}}-E_{\mathrm{d}}\right)$ & $\mathrm{EMF}=(-5 \mathrm{SOC}+5) E_{\mathrm{c}}+(5 \mathrm{SOC}-4) E_{\mathrm{d}}$ \\
\hline
\end{tabular}

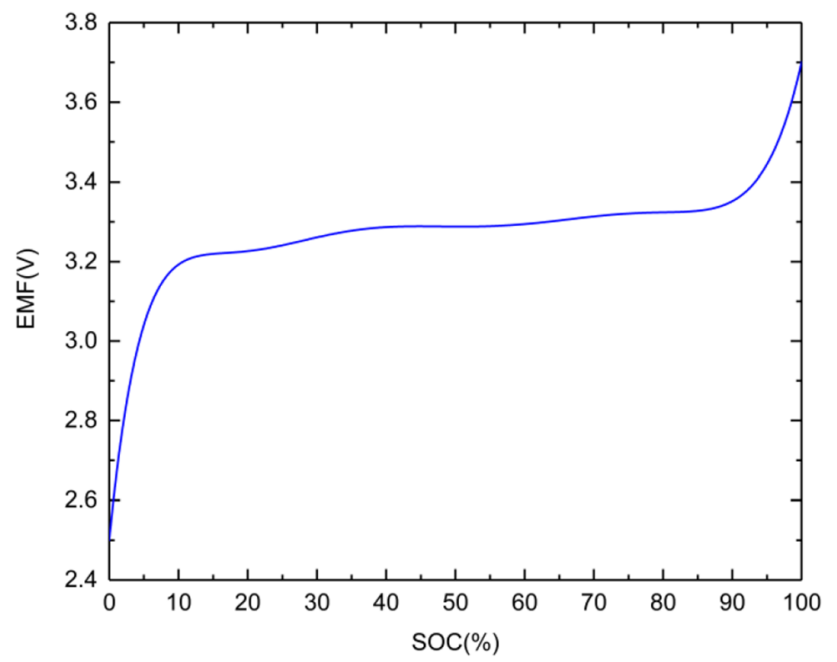

Fig. $4 \mathrm{LiFePO}_{4}$ battery EMF curve

\subsubsection{Parameter Estimation of Equivalent Impedance}

The hybrid pulse power characterization (HPPC) test was carried out according to the Freedom CAR Battery Test Manual. The discharge current and voltage responses were recorded every $0.5 \mathrm{~s}$ to determine model parameters. Table 3 shows the pulse current of an HPPC test. Figure 5 shows the voltage response curve corresponding to pulse discharge.

From the HPPC test voltage curve, it can be concluded that:

At the beginning of pulse discharge, the instantaneous vertical drop in voltage is caused by the ohmic internal resistance, so the internal resistance can be obtained via

$R_{0}=\Delta U_{1} / I$

where $\Delta U_{1}$ is the voltage caused by the ohmic internal resistance at the moment of discharge and $I$ is the discharge current.

After the pulse discharge, the battery terminal voltage rises slowly, which is the voltage rebound. This is equivalent to the zero-input response of the RC network. From this, the following is obtained:

Table 3 HPPC pulse current
$\Delta U_{2}=U_{1}+U_{2}+U_{3}=U_{01} \mathrm{e}^{-\frac{t}{\tau_{1}}}+U_{02} \mathrm{e}^{-\frac{t}{\tau_{2}}}+U_{03} \mathrm{e}^{-\frac{t}{\tau_{3}}}$

where $\Delta U_{2}$ is the total rebound voltage; $U_{01}, U_{02}$ and $U_{03}$ are the initial polarization voltages; and $\tau_{1}, \tau_{2}$ and $\tau_{3}$ are the time constants of RC networks ( $\tau=\mathrm{RC}$ ). The parameters $U_{01}, U_{02}$, $U_{03}, \tau_{1}, \tau_{2}$ and $\tau_{3}$ need to be determined. The experimental voltage data are fitted with MATLAB to obtain $\tau_{1}, \tau_{2}$ and $\tau_{3}$.

During the pulse discharge, the slow voltage drop is affected by the battery polarization. The polarization voltage is equivalent to the zero-state response of the RC network, thereby yielding

$U(t)=U^{\prime}-I R_{1}\left(1-\mathrm{e}^{-\frac{t}{\tau_{1}}}\right)-I R_{2}\left(1-\mathrm{e}^{-\frac{t}{\tau_{2}}}\right)-I R_{3}\left(1-\mathrm{e}^{-\frac{t}{\tau_{3}}}\right)$

where $U(t)$ is the voltage at any time during the voltage drop; $U^{\prime}$ is the starting point after the voltage vertical drop and $R_{1}$, $R_{2}$ and $R_{3}$ are the parameters to be determined. The experimental voltage data are fitted with MATLAB to obtain $R_{1}$, $R_{2}$ and $R_{3}$. Further, the polarization capacitances $C_{1}, C_{2}$ and $C_{3}$ can be obtained via $\tau_{1}=R_{1} C_{1}, \tau_{2}=R_{2} C_{2}$ and $\tau_{3}=R_{3} C_{3}$.

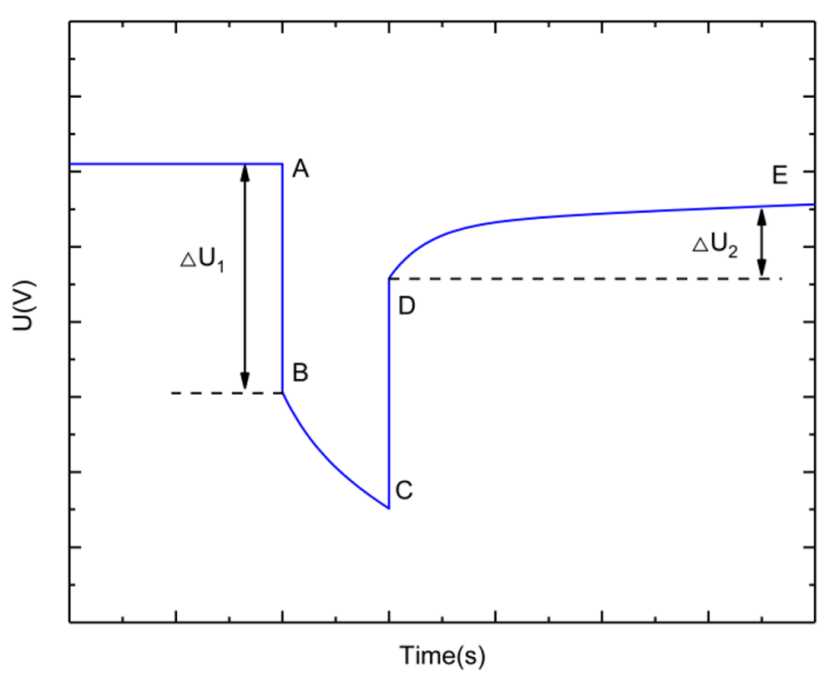

Fig. 5 Voltage response during the HPPC test

\begin{tabular}{lllllllll}
\hline Time node/s & 0 & 0 & 10 & 10 & 50 & 50 & 60 & 60 \\
\hline Current/A & 0 & 2.2 & 2.2 & 0 & 0 & -2.2 & -2.2 & 0 \\
\hline
\end{tabular}


According to the above, all the model parameters can be calculated:

$R_{0}=0.03 \Omega, R_{1}=0.003 \Omega, C_{1}=43,000 \mathrm{~F}, R_{2}=0.0035 \Omega$, $C_{2}=50,000 \mathrm{~F}, R_{3}=0.011 \Omega$, and $C_{3}=49,900 \mathrm{~F}$.

\section{Simulation and Analysis Based on Battery and Vehicle Models}

\subsection{Battery Simulation Analysis}

The model was simulated in Urban Dynamometer Driving Schedule (UDDS) working conditions to verify its accuracy. UDDS working conditions were designed by the U.S. Environmental Protection Agency for light vehicles [19]. Figure $6 \mathrm{a}$ shows the battery current as a function of time. The discharge current is the input signal, and the terminal voltage response is the output. The simulated and measured voltage signals are compared in Fig. 6b, where the blue curve is the measured voltage and the red is the simulation. Figure $6 \mathrm{c}$ shows the error changes of simulated and measured voltage under UDDS.

As shown in Fig. 6c, the error between the simulated and the measured voltage is small. The maximum error is $1.78 \%$, and the average error is $0.23 \%$. These results show that the model can accurately capture the battery dynamics.

\subsection{Vehicle Simulation Analysis}

To further verify the reliability and practicability of the battery model, it was used to simulate a whole electric vehicle.

\subsubsection{Modelica Model of Electric Vehicle}

The whole vehicle is divided into the power system module, mechanical component module, control module and other accessory modules by means of modular division. The power system module includes the battery pack and motor system. The battery pack is based on the $\mathrm{LiFePO}_{4}$ battery model established in this paper. The gain module in the Modelica Standard Library is used to form the series-parallel connections required for the battery. The motor system model is based on the permanent magnet synchronous motor (PMSM), which integrates and encapsulates the built motor model and the motor controller. The mechanical component module includes the powertrain, brakes and chassis with wheel and suspension. The powertrain model is based on the vehicle transmission model, which transmits the motor signal to the wheel and suspension of the vehicle chassis through the defined interface under vehicle control. The control module mainly includes the driver control and vehicle control models. The vehicle control model is the key to simulating the vehicle because it realizes input of simulation

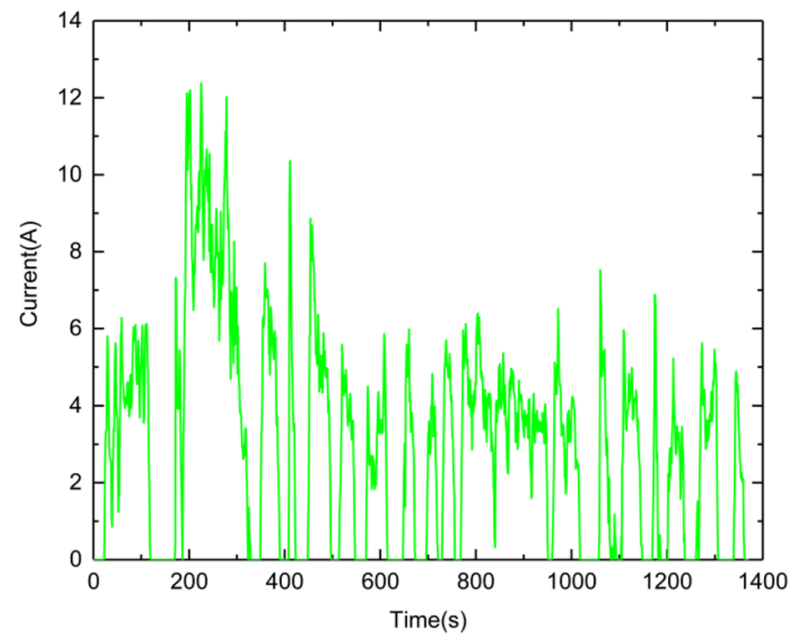

(a) Battery current under UDDS

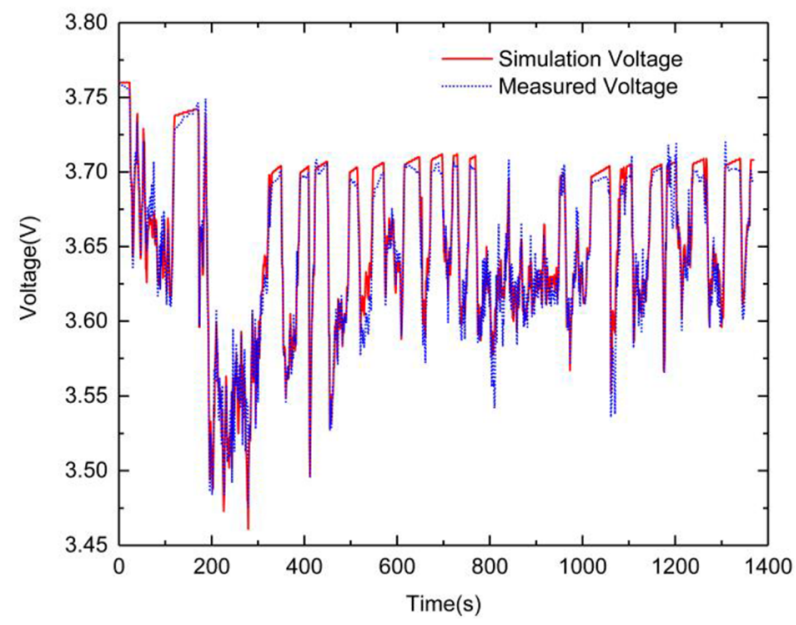

(b) Comparison of simulated and measured battery terminal voltages

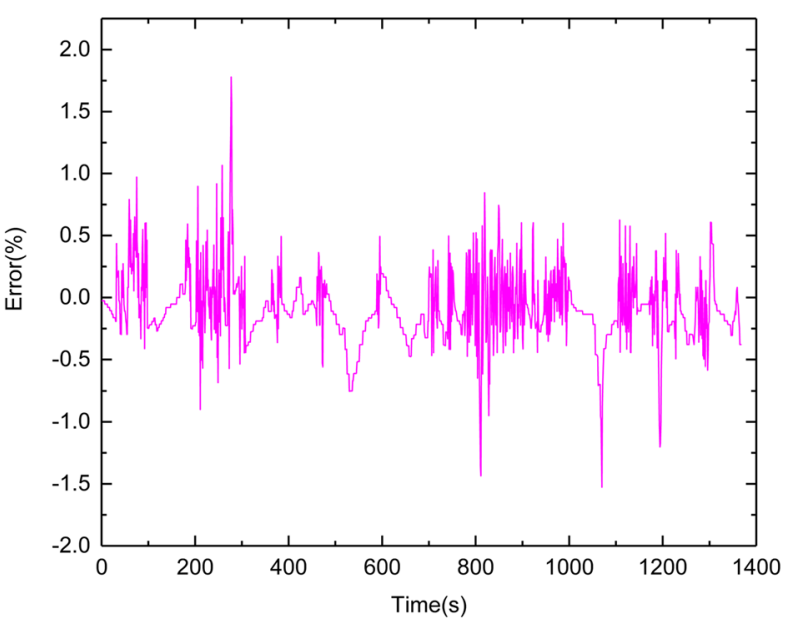

(c) Error of simulated and measured battery terminal voltages

Fig. 6 Simulation results under UDDS 


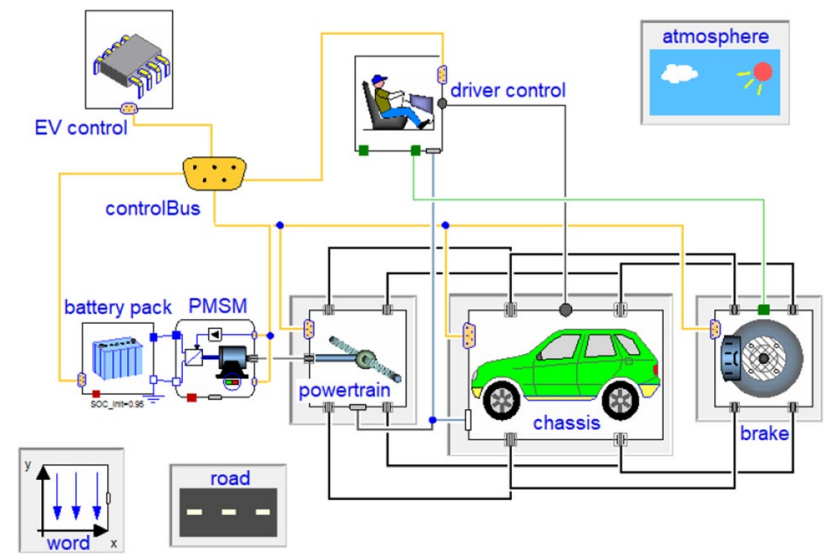

Fig. 7 EV simulation model

parameters and output of simulation data. The accessory module includes the word model, atmospheric model and road model. The word model mainly provides parameters such as gravitational acceleration for the entire vehicle. The road model mainly describes the basic road information and gives the cyclic road condition in simulation, which is used to simulate the road resistance when the vehicle is running. The atmospheric model mainly refers to the external environmental conditions set when simulating a pure EV, including such factors as the air resistance exerted on the vehicle. Finally, the models were coupled and connected through the defined interfaces between different subject areas, and the multi-domain model of a pure EV was established in MWorks, as shown in Fig. 7. The parameters of the other components are taken from the literature [20-22]. Some simulation parameters are shown in Table 4.

\subsubsection{Simulation Analysis of Driving Conditions}

The vehicle model was simulated under New European Driving Cycle (NEDC) to verify the accuracy of the new battery model. By analyzing the NEDC of the simulation, the battery voltage, current, SOC and vehicle mileage are obtained, as shown in Fig. 8.

Figure $8 \mathrm{a}$, b shows the battery voltage and current curves, respectively, when the vehicle is simulated under NEDC. At the beginning, the battery SOC was set to $95 \%$, at which time the voltage was $346.65 \mathrm{~V}$. At the end of the simulation, the voltage dropped to $336.99 \mathrm{~V}$. When the current is positive, the car is accelerating or at constant speed. When the current is negative, the car is in regenerative braking condition, and the battery pack is charged. The trends of the whole voltage and current agree with the dynamic theory of EVs.

As seen in Fig. 8c, the battery SOC decreases from 95 to $86 \%$ after the simulation. From Fig. 8d, the driving mileage of the whole simulation is calculated to $10.99 \mathrm{~km}$, which is $0.06 \mathrm{~km}(0.54 \%)$ different from the theoretical driving mileage of $11.05 \mathrm{~km}$. The energy consumption of the whole process is estimated to be $172 \mathrm{Wh} / \mathrm{km}$. The results show that the established battery model has high accuracy and can be used for vehicle simulation tests.

\section{Conclusions}

By analyzing the dynamics of a $\mathrm{LiFePO}_{4}$ battery for an $\mathrm{EV}$, the influence of hysteresis voltage on model accuracy has been considered. A third-order RC network was used to better simulate the polarization inside the battery, and an improved battery model was established by using Modelica. The battery simulation under UDDS was implemented on the MWorks platform. The difference between the simulated and measured voltages is small, with a maximum error of $1.78 \%$, average error of $0.23 \%$. Subsequently, the whole EV system was simulated on the MWorks platform combined with the vehicle model, and the difference between the simulated and theoretical
Table 4 Partial list of EV model parameters

\begin{tabular}{llll}
\hline Simulation parameters & Value & Simulation parameters & Value \\
\hline Maximum voltage of battery (V) & 370 & Rated power of motor $(\mathrm{kW})$ & 30 \\
Nominal voltage of battery (V) & 320 & Rated speed of motor (rpm) & 2800 \\
Nominal capacity of battery (Ah) & 66 & Peak speed of motor (rpm) & 6000 \\
Nominal energy of battery (KWh) & 20.15 & Rated torque of motor $(\mathrm{Nm})$ & 100 \\
Curb weight $(\mathrm{kg})$ & 1300 & Peak torque of motor $(\mathrm{Nm})$ & 200 \\
Full mass $(\mathrm{kg})$ & 1800 & Rated voltage of motor $(\mathrm{V})$ & 312 \\
Frontal area $\left(\mathrm{m}^{2}\right)$ & 2.0 & Peak voltage of motor $(\mathrm{V})$ & 400 \\
Wind resistance coefficient & 0.4 & Rated current of motor $(\mathrm{A})$ & 120 \\
Rolling friction coefficient & 0.015 & Transmission efficiency & 0.90 \\
\hline
\end{tabular}




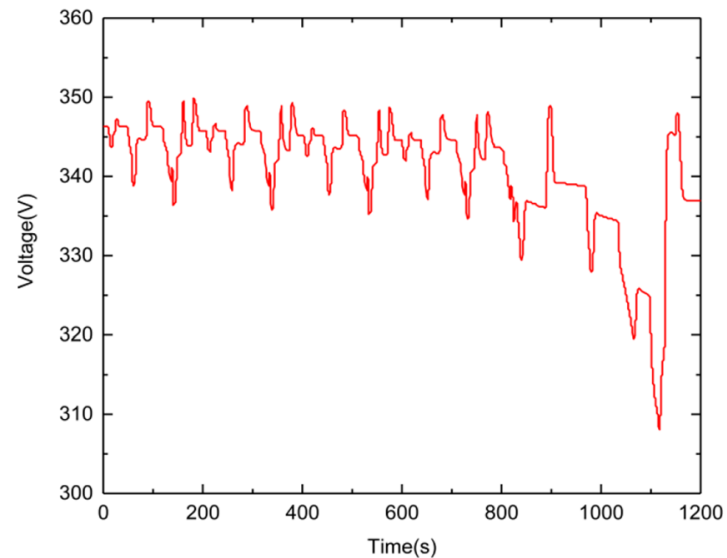

(a) Battery voltage

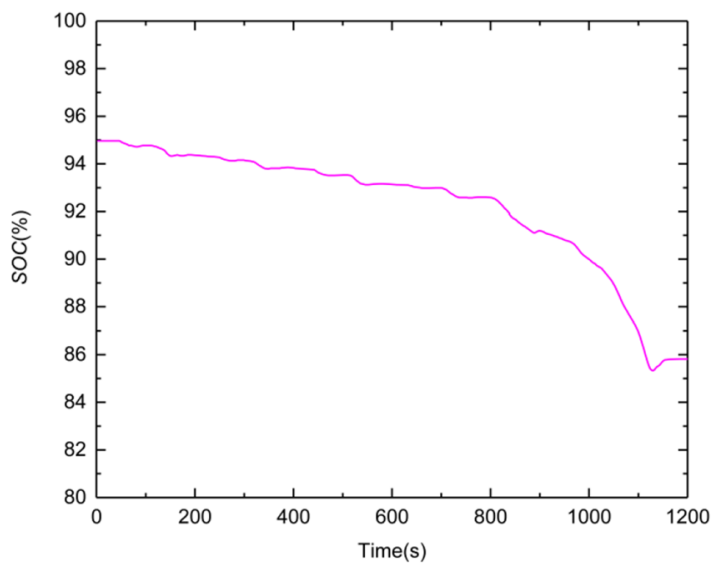

(c) Battery SOC

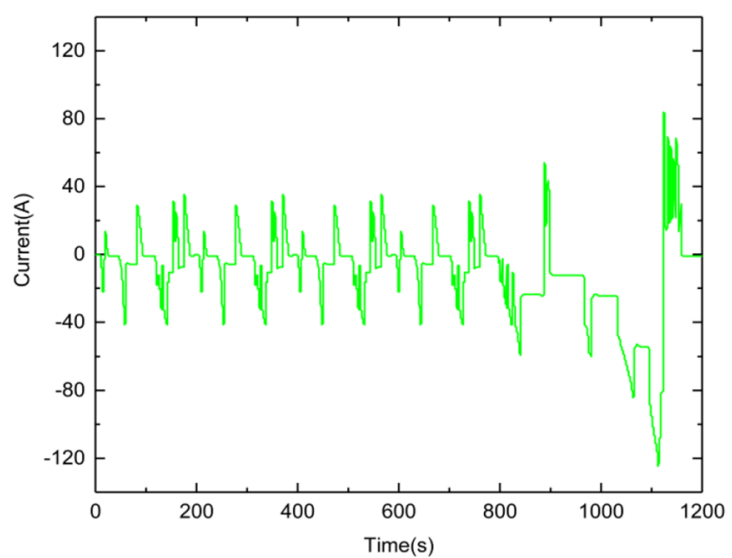

(b) Battery current

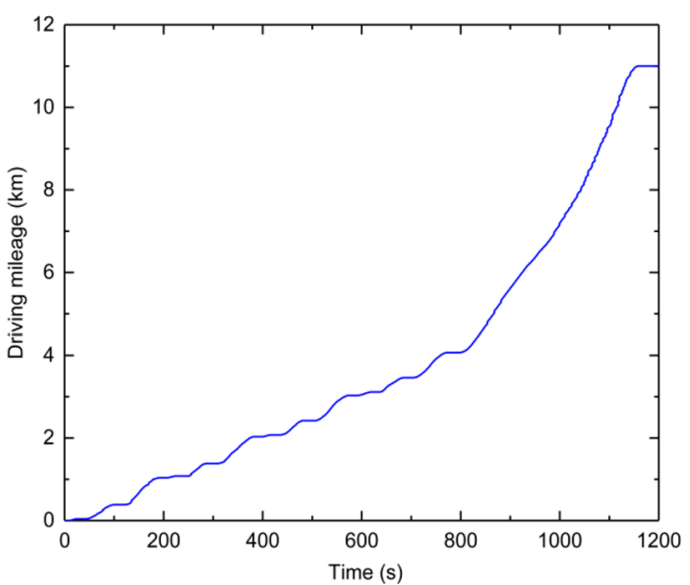

(d) Vehicle driving mileage

Fig. 8 Vehicle simulation results under NEDC

results is $0.54 \%$. This further verifies the effectiveness and practicability of the battery model. In conclusion, the third-order RC battery model with hysteretic voltage better reflects the dynamics of the $\mathrm{LiFePO}_{4}$ battery. This battery model can be used to simulate EVs, providing a reference for unified modeling and simulation of their multi-domain systems. Later research will take into account the dynamic relationship between battery model parameters and SOC and consider the influence of temperature, so as to establish a multi-factor battery model, which will further improve the model accuracy.

Acknowledgements This work was supported by the National Key Research and Development Program of China (No. 2018YFB0106204-03)

\section{Compliance with Ethical Standards}

Conflict of interest On behalf of all authors, the corresponding authors state that there is no conflict of interest.
Open Access This article is distributed under the terms of the Creative Commons Attribution 4.0 International License (http://creativeco mmons.org/licenses/by/4.0/), which permits unrestricted use, distribution, and reproduction in any medium, provided you give appropriate credit to the original author(s) and the source, provide a link to the Creative Commons license, and indicate if changes were made.

\section{References}

1. Wang, J.L., Wu, Y.Z., Xiong, H.Y.: Modeling and simulation of pure electric vehicle. Comput. Simul. 32(4), 190-195 (2015)

2. Elmqvist, H., Mattsson, S.E., Otter, M.: Modelica-a language for physical system modeling, visualization and interaction. Paper presented at the 1999 IEEE International Symposium on Computer Aided Control System Design, Kohala Coast-Island of Hawai'i, 27 August 1999

3. Li, Z.H., Zhang, H.L., Zheng, L.: Description of PDE models in Modelica. Paper presented at the 2008 International Symposium on Computer Science and Computational Technology, Shanghai, 20-22 December 2008

4. Sven, E.M., Hilding, E., Martin, O.: Physical system modeling with Modelica. Control Eng. Pract. 6(4), 501-510 (1998) 
5. Cheng, K.W.E., Divakar, B.P., Wu, H.J., et al.: Battery management system (BMS) and SOC development for electrical vehicles. IEEE Trans. Veh. Technol. 60(1), 76-88 (2011)

6. Hu, X.S., Li, S.B., Peng, H.: A comparative study of equivalent circuit models for Li-ion batteries. J. Power Sources 198, 359-367 (2012)

7. Sung, W., Shin, C.B.: Electrochemical model of a lithium-ion battery implemented into an automotive battery management system. Comput. Chem. Eng. 76, 87-97 (2015)

8. Pramanik, S., Anwar, S.: Electrochemical model based charge optimization for lithium-ion batteries. J. Power Sources 313, 164-177 (2016)

9. Smith, K.A., Rahn, C.D., Wang, C.Y.: Control oriented 1D electrochemical model of lithium ion battery. Energy Convers. Manag. 48(9), 2565-2578 (2007)

10. O’Gorman, C.C., Ingersoll, D., Jungst, R.G., et al.: Artificial neural network simulation of battery performance. Paper presented at the 31st Annual Hawaii International Conference on System Sciences. Kohala Coast-Island of Hawai'i, 9 January 1998

11. Johnson, V.H.: Battery performance models in ADVISOR. J. Power Sources 110(2), 321-329 (2002)

12. Zhang, Z.Q., Wu, Z.B., Weng, R.C.: Simulation research on model of $\mathrm{LiFePO}_{4} \mathrm{Li}$-ion power battery. Automob. Appl. Technol. 41(1), 93-95 (2016)

13. Huang, X.C., Zong, Z.J., Chen, C.H., et al.: Modeling and simulation of power battery for electric vehicle based on Modelica. Comput. Eng. Des. 33(5), 2073-2077 (2012)

14. García-Plaza, M., Serrano-Jiménez, D., Eloy-García Carrasco, J., et al.: A Ni-Cd battery model considering state of charge and hysteresis effects. J. Power Sources 275, 595-604 (2015)
15. Eichi, H.R., Chow, M.Y.: Modeling and analysis of battery hysteresis effects. In: Proceedings paper presented at the 2012 IEEE Energy Conversion Congress and Exposition, Raleigh, NC, 15-20 September 2012

16. Federico, B., Nicola, F., Roberto, S., et al.: Hysteresis modeling in Li-ion batteries. IEEE Trans. Magn. 50(11), 1-4 (2014)

17. Tan, X.J.: Design of Power Battery Management System for Electric Vehicle. Sun Yat-sen University Press, Guangzhou (2011)

18. Lee, S.J., Kim, J.H., Lee, J.M., et al.: The state and parameter estimation of a Li-ion battery using a new OCV-SOC concept. Paper presented at the 2007 IEEE Power Electronics Specialists Conference, Orlando, FL, 17-21 June 2007

19. Fredette, D., Pavlich, C., Ozguner, U.: Development of a UDDScomparable framework for the assessment of connected and automated vehicle fuel saving techniques. IFAC-PapersOnLine 45(15), 306-312 (2015)

20. Standardization Administration of China: GB/T 18385-2005. Electric Vehicles-Power Performance-Test Method. China Standards Press, Beijing (2005)

21. Standardization Administration of China: GB/T 18386-2005. Electric Vehicles-Energy Consumption and Range-Test Procedures. China Standards Press, Beijing (2017)

22. Cheng, L., Qin, D.C., Wang, Y.K., et al.: Modeling and simulation of pure electric bus based on Modelica. Automob. Technol. 48(8), 43-48 (2017) 